\title{
Effect of Clustering on the Heat Generated by Superparamagnetic Iron Oxide Nanoparticles
}

\author{
Marco Lattuada*
}

\begin{abstract}
Magnetic nanoparticles have been the subject of enormous investigations for their potential use as cancer treatment via hyperthermia. This is due to their ability to generate heat when exposed to an external magnetic field oscillating at sufficiently high frequency. There are many different parameters that need to be considered when designing the optimal nanoparticle formulation for hyperthermia. The effect of the formation of clusters of nanoparticles, which is either an unwanted side effect of poor colloidal stability of the particles, or a desired formulation strategy, has poorly understood consequences on the amount of heat generated by the nanoparticles. The objective of this work is to address this problem from the theoretical side by performing detailed simulations to investigate the effect of incorporation of magnetic nanoparticles in clusters on the amount of heat generated as a function of the particle size, cluster size and particles magnetic properties.
\end{abstract}

Keywords: Clusters · Hyperthemia · Langevin simulations · Superparamagnetic iron oxide nanoparticles (SPIONs)

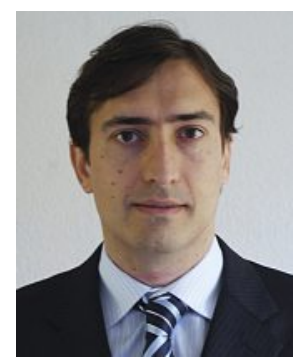

Marco Lattuada received a master's degree in Chemical Engineering from Politecnico di Milano, Italy. He obtained a $\mathrm{PhD}$ in Chemical Engineering from ETH Zurich, under the guidance of Prof. Massimo Morbidelli. He then moved to MIT, where he carried out a postdoc for over two years in Prof. Alan Hatton's group. Afterwards, he moved back to ETH Zurich, where he worked as a senior scientist and group leader for about six years, before eventually moving to the University of Fribourg, at the Adolphe Merkle Institute, where he obtained a prestigious Swiss National Science Foundation Professor-founded professorship, to start his independent career. He is currently a Professor in the Department of Chemistry, at the University of Fribourg. His research activity is a balanced combination of experimental and modeling work, aiming at engineering smart nanoand micro-particles, and at exploiting their self-assembly, with the objective to design novel soft materials.

\section{Introduction}

Hyperthermia is one the most promising strategies under investigation to combat certain types of cancer.[1] Instead of relying on the use of drugs or radiations to kill cancer cells, hyperthermia makes use of the heat generated by nanoparticles exposed to a external stimulus. ${ }^{[1,2]}$ Typically, this stimulus is either an electromagnetic radiation, as in the case of gold nanoparticles, ${ }^{[1]}$ or a high frequency alternating magnetic field, as in the case of superparamagnetic nanoparticles. ${ }^{[3]}$ The latter, more specifically superparamagnetic iron oxide nanoparticles (SPIONs), have already been used in clinical trials and hold great promise for clinical use. ${ }^{[4]}$ Many hurdles are still to be overcome to optimize the performance of SPIONs. Rational design of SPIONs

\footnotetext{
${ }^{*}$ Correspondence: Prof. M. Lattuada

E-mail: marco.lattuada@unifr.ch

Department of Chemistry, University of Fribourg, Chemin du Musée 9,

$\mathrm{CH}-1700$ Fribourg
}

configuration is probably one of the most urgent problems to solve. Particle size, colloidal stability and crystalline properties are the most important parameters affecting the performance of SPIONs. ${ }^{[3]}$ The control of particle size has been achieved by introducing high temperature organic synthesis of magnetite nanoparticles, ${ }^{[5]}$ which have overcome the limitations of the much simpler and previously used coprecipitation method. ${ }^{[6]} \mathrm{A}$ plethora of strategies have been developed to functionalize the surface of SPIONs, which is a necessary step, because particles prepared via high temperature organic synthesis are usually hydrophobic (i.e. coated with oleic acid), and thus not suitable to be used as such for hyperthermia application, ${ }^{[2,3]}$ and to improve their crystallinity. In fact, some standard synthetic routes produce particles with poor crystallinity, or with the wrong crystalline phases (such as wüstite), which lead to poor magnetic properties. ${ }^{[7]}$

The effect of the colloidal stability of nanoparticles is also of great relevance, and it is fair to claim that it is probably the least well understood factor affecting the amount of heat generated by SPIONs, and arguably the most difficult to control. ${ }^{[8]}$ The formation of aggregates is one of the consequences of poor colloidal stability of SPIONs. ${ }^{[9]}$ This uncontrolled aggregation can have potentially deleterious effects on hyperthermia. On the flip side, many synthetic and functionalization routes have been developed that lead to the formation of clusters with controlled size. ${ }^{[10]}$

From the theoretical viewpoint, several studies have been published presenting models to predict the amount of heat generated by nanoparticles. Linear response theory (LRT), developed by Rosensweig in 2002,[11] is the most successful theory. It does have some limitations though, as it is only applicable to spherical particles with a magnetization that depends linearly on the applied magnetic field. In the case of clusters, though, not many investigations have been published. The only feasible approach to simulate the behavior of clusters of spherical particles is to use Langevin simulations, ${ }^{[12]}$ which can account for magnetic interactions among particles, and for relaxation of particles due to both Brownian and Neel mechanisms. Therefore, the question that this paper is addressing is the following: how does the incorporation of SPIONs into clusters affect their heat generation capability? 
We have used Langevin-based simulations to answer this very important question.

\section{Simulation Strategy}

In this work, Langevin-type simulations, accounting for all relevant physical phenomena occurring during magnetic relaxation have been used to compute the heat dissipated by a spherical particle, both isolated and as part of a cluster. ${ }^{[12]}$ All particles are spherical in shape and are assumed to possess only one magnetic domain, with a magnetocrystalline anisotropy described by means of the Stoner-Wohlfarth model.[13] Two unit vectors are required to follow the direction of easy axis of magnetization, and the magnetic moment. The energy of a magnetic particle with a magnetic moment $m=\mu_{0} M_{s} \cdot V_{p}$, with $M_{s}$ being the saturation magnetization of the particle, $V_{p}=4 / 3 \cdot \pi \cdot \mathrm{R}_{\mathrm{p}}{ }^{3}$ its magnetic volume and $\mu_{0}$ the vacuum magnetic permeability, with the magnetic moment having the orientation $\mathbf{e}$ in the presence of an external magnetic field $\mathbf{H}$, and with the easy axis of magnetization identified by the vector $\mathbf{n}$ is given by the following expression:

$$
E=-m \mathbf{e} \cdot \mathbf{H}(t)-K_{V} V_{P}(\mathbf{e} \cdot \mathbf{n})^{2}
$$

$K_{V}$ is the magnetocrystalline anisotropy constant. The mathematical model implemented has been proposed by Stepanov and Shliomis. ${ }^{[12,14]}$ Accordingly, to model the relaxation behavior of a particle, we need to solve two vector differential equations to compute the time evolution of the unit vectors $\mathbf{e}$ and $\mathbf{n}$. The first one describes the change in orientation of the unit vector n, caused by a random contribution due to rotational diffusion, and by a deterministic contribution due to the magnetic torque. In the case of a cluster, which is a rigid body, similar considerations apply, as one needs to keep track of the orientation of the magnetic moments and of the easy axes of magnetization of each particle. Additionally, the magnetic torque is given by the sum of all contributions of all magnetic moments. Because all particles are incorporated in a single cluster, which rotates as a rigid body, all easy axes of magnetization of all its particles will undergo the same external rotation. If we consider $N$ particles with unit dipole vectors $\mathbf{e}_{i}$ and easy axis of magnetization $\mathbf{n}_{i}$, we have:

$$
\frac{d \mathbf{n}_{j}}{d t}=\left(\frac{m}{6 \eta V_{H}}\left(\sum_{i=1}^{N} \mathbf{e}_{i} \times \mathbf{H}(t)\right)+\boldsymbol{\Omega}_{\text {rand }, e}\right) \times \mathbf{n}_{j}=\boldsymbol{\Omega} \times \mathbf{n}_{j}
$$

In Eqn. (2), $\eta$ is the viscosity of the solvent in which particles are moving, $V_{H}$ is the hydrodynamic volume of the cluster, or of the particle in the case of one single particle. The hydrodynamic volume of a single particle accounts not only for the volume of the particle core, but also for the volume of the shell, usually consisting of small ligands or of a polymer layer, with known thickness, stabilizing the surface of the particles. In this work, it has been assumed that a shell with a thickness of one nanometer, roughly corresponding to one layer of oleic acid molecules, surrounds each particle. The hydrodynamic radius of the cluster has been estimated by means of Kirkwood-Rieseman theory, ${ }^{[15]}$ because it depends not only on the size and number of particles in each cluster, but also on their relative positions. $\boldsymbol{\Omega}_{\text {rand,e }}$ is the external random motion contribution to the angular velocity. Eqn. (2) implements the so-called Landau-Lifshitz model and describes the change in orientation of the magnetic moment. ${ }^{[14]}$ This includes not only the contribution of the magnetic torque, but also two random contributions, and the energy penalty due to the misalignment of the magnetic moment with easy axis of magnetization, in accordance to Stoner-Wohlfarth's model:

$$
\frac{d \mathbf{e}_{j}}{d t}=\left[\frac{m}{6 \eta V_{H}}\left(\sum_{i=1}^{N} \mathbf{e}_{i} \times \mathbf{H}(t)\right)+\frac{m}{2 \tau_{0} K_{V} V_{P}}\left(\mathbf{e}_{j} \times\left(\mathbf{H}_{j}(t)+\frac{2 K_{V} V_{P}}{m}\left(\mathbf{e}_{j} \cdot \mathbf{n}_{j}\right) \mathbf{n}_{j}\right)\right)\right] \times \mathbf{e}_{j}+\left(\boldsymbol{\Omega}_{r n a d, e}+\boldsymbol{\Omega}_{r a n d, i, j}\right) \times \mathbf{e}_{j}
$$

In Eqn. (3) there are two random contributions: one external (due to rotational diffusivity, the same appearing in Eqn. (2)), and the other one internal, related to the internal random fluctuations of the magnetic moment vector. In all simulations, it is assumed that the applied magnetic field has a cosinusoidal time dependence:

$$
\mathbf{H}(t)=\mathbf{H}_{0} \cos (2 \pi f \cdot t)
$$

with frequency $f$, and external field $\mathbf{H}_{0}$ oriented along the $z$ direction. In the case of clusters, magnetic dipolar interactions need to be considered, which are assumed to be equivalent of those generated by point dipoles centered at the particles centers. The total magnetic field that each particle feels is then given by the sum of the external field and the sum of the dipolar fields exerted by all other particles in the system:

$$
\mathbf{H}_{j}(t)=\mathbf{H}(t)+\frac{1}{4 \pi} \sum_{i=1 \neq j}^{N}\left(\frac{3 \mathbf{r}_{i j}\left(\mathbf{m}_{i} \cdot \mathbf{r}_{i j}\right)}{r_{i j}^{5}}-\frac{\mathbf{m}_{i}}{r_{i j}^{3}}\right)
$$

Eqns (2) and (3) need to be discretized to be solved. The algorithm proposed by Makino and Doi has been used to integrate numerically the equations. ${ }^{[16]}$ Explicitly, Eqn. (2) becomes:

$$
\begin{aligned}
& \Delta \boldsymbol{\Theta}_{B}(t)=\frac{m}{6 \eta V_{H}}(\mathbf{e} \times \mathbf{H}(t)) \Delta t+\sqrt{\frac{\Delta t}{\tau_{B}}} \mathbf{R}_{e} \\
& \Delta \mathbf{n}=\Delta \boldsymbol{\Theta}_{B} \times \mathbf{n}-\frac{1}{2}\left(\mathbf{n}\left(\Delta \Theta_{B}(t) \cdot \Delta \Theta_{B}(t)\right)-\Delta \Theta_{B}(t)\left(\Delta \Theta_{B}(t) \cdot \mathbf{n}\right)\right)
\end{aligned}
$$

where $\mathbf{R}_{e}$ is a random vector with normally distributed components having unit variance and zero mean, and the Brownian relaxation time $\tau_{B}$ is defined as:

$$
\tau_{B}=\frac{3 \eta V_{H}}{k T}
$$

with $k$ being the Boltzmann constant and $T$ the absolute temperature. The equivalent discrete version of Eqn. (3) is the following:

$$
\begin{aligned}
& \Delta \boldsymbol{\Theta}_{N}(t)=\left[\frac{m}{6 \eta V_{H}}(\mathbf{e} \times \mathbf{H}(t))+\frac{m}{2 \tau_{0} K_{V} V_{P}}\left(\mathbf{e} \times\left(\mathbf{H}(t)+\frac{2 K_{V} V_{P}}{m}(\mathbf{e} \cdot \mathbf{n}) \mathbf{n}\right)\right)\right] \Delta t+\left(\sqrt{\frac{\Delta t}{\tau_{B}}} \mathbf{R}_{e}+\sqrt{\frac{\Delta t}{\tau_{D}}} \mathbf{R}_{i}\right) \\
& \Delta \mathbf{e}=\Delta \boldsymbol{\Theta}_{N} \times \mathbf{e}-\frac{1}{2}\left(\mathbf{e}\left(\Delta \boldsymbol{\Theta}_{N}(t) \cdot \Delta \boldsymbol{\Theta}_{N}(t)\right)-\Delta \boldsymbol{\Theta}_{N}(t)\left(\Delta \boldsymbol{\Theta}_{N}(t) \cdot \mathbf{e}\right)\right)
\end{aligned}
$$

$\mathbf{R}_{i}$ is another random vector with normally distributed components having unit variance and zero mean, and the internal magnetic relaxation time $\tau_{D}$, related to Neel relaxation mechanism, is defined as:

$$
\tau_{D}=\tau_{0} \frac{K_{V} V_{p}}{k T}
$$


where $\tau_{0}$ is a characteristic time, usually set equal to about $10^{-9}$ $\mathrm{s}$, which includes the effect of the internal magnetic viscosity responsible for internal relaxation. ${ }^{[11]}$ The time step in simulations is chosen as the 1/100 of the smallest time scale in the simulation, namely:[12]

$$
\Delta t=\frac{1}{100} \min \left\{\frac{1}{f}, \tau_{D}, \tau_{B}, \frac{6 \eta V_{H}}{m H_{0}}, \frac{2 \tau_{0} K_{V} V_{P}}{m H_{0}+K_{V} V_{P}}\right\}
$$

All simulations have been performed with a sufficient number of time steps, such that at least 10 full cycles have been covered. After each simulation step, the unit vectors $\mathbf{e}_{i}$ and $\mathbf{n}_{i}$ are renormalized to prevent unphysical results. It should be noted that also the positions of all particles inside the clusters need to be updated using the following equation:

$$
\frac{d \mathbf{r}_{i}}{d t}=\mathbf{\Omega} \times \mathbf{r}_{i}
$$

In Eqn. (11), the angular velocity $\boldsymbol{\Omega}$ is the same as in Eqn. (2). The power per unit mass of particles generated by dissipation due to the hysteresis of the rapidly oscillating magnetic dipole value is obtained from the integral of the magnetization with the applied magnetic field over one cycle:[11]

$$
P=-f \mu_{0} \int \mathbf{M} \cdot d \mathbf{H}=\frac{2 \pi f^{2} m}{N \rho_{P} V_{P}} \int_{0}^{\frac{1}{f}} \sum_{i=1}^{N} \mathbf{e}_{i} \cdot \mathbf{H}_{0} \sin (2 \pi f \cdot t) d t
$$

A list of the values of parameters used in the simulations is shown in Table 1.

Table 1. List of parameter values used in the simulations

\begin{tabular}{c|c}
\hline Parameter & Value \\
\hline$R_{\rho}$ & $1-15 \mathrm{~nm}$ \\
\hline$K_{V}$ & $10^{3}-4 \cdot 10^{4} \mathrm{~J} / \mathrm{m}^{3}$ \\
\hline$R_{H}$ (of one particle) & $R_{p}+1 \mathrm{~nm}$ \\
\hline$N$ & $1,2,19,87$ \\
\hline$f$ & $10^{5}-5.5 \cdot 10^{5} \mathrm{~Hz}$ \\
\hline$\tau_{0}$ & $10^{-9} \mathrm{~s}$ \\
\hline$\eta($ water $)$ & $0.000879 \mathrm{~Pa} \cdot \mathrm{s}$ \\
\hline$T$ & $298.15 \mathrm{~K}$ \\
\hline$M_{s}$ & $2.9 \cdot 10^{5} \mathrm{~A} / \mathrm{m}$ \\
\hline$H_{0}$ & $2 \cdot 10^{4} \mathrm{~A} / \mathrm{m}$ \\
\hline$\rho_{\rho}$ & $5180 \mathrm{Kg} / \mathrm{m}^{3}$ \\
\hline
\end{tabular}

\section{Results and Discussion}

The objective of the simulations is to provide insight on the amount of heat generated by magnetic nanoparticles, commonly referred to as the specific adsorption rate (SAR), considering magnetite as material, and to understand the effect of incorporation of particles into clusters on SAR values. Several parameters have been varied in the simulations: the frequency of the applied magnetic field, the nanoparticle size, the number of particles in the cluster and the magnetocrystalline anisotropy constant. The frequency of the magnetic field typically varies between 100 and 500 $\mathrm{kHz}$, and has a strong effect on the heat generated by the nanoparticles. ${ }^{[11]}$ The nanoparticle size also plays a crucial role and is one of the factors used to engineer optimal formulations for hyperthermia. ${ }^{[1]}$ Since it is known that magnetite nanoparticles become ferromagnetic above a size of about $25-30 \mathrm{~nm},{ }^{[4]}$ the nanoparticle diameter has been changed between 2 and $30 \mathrm{~nm}$. The number of nanoparticles in the cluster was chosen to understand the interplay between their interactions and cluster size on the SAR values. The magnetocrystalline anisotropy constant determines the importance of anisotropy energy, and its value is difficult to determine experimentally and varies strongly depending on the preparation method used to synthesize magnetic nanoparticles, going from the bulk value of magnetite, which is rather high, to that of maghemite, which is very small. ${ }^{[9]}$ To understand the effect of clustering on the SAR values of nanoparticles, the behavior of single particles has been simulated first, followed by dumbbells of particles, and then by clusters with two different number of particles, i.e. 19 and 87 , referred to as small and large clusters, respectively. Both clusters structures have been generated assuming particles to be arranged in a hexagonal compact structure.

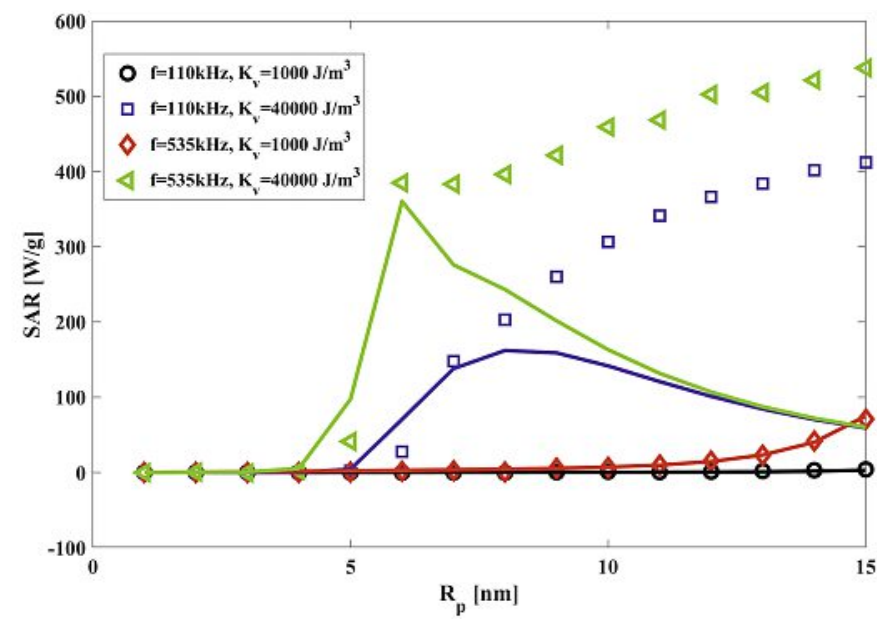

Fig. 1. SAR values of single SPIONs as a function of the particle radius, for the frequencies and magnetocrystalline anisotropy constants shown in the legend. Points are the results of the Langevin simulations, while lines are predictions from linear response theory.

Fig. 1 shows the calculated SAR values for single particles, as function of particle size, for two different frequency values $(110 \mathrm{kHz}$, and $535 \mathrm{kHz}$ ) and two different values of the magnetocrystalline anisotropy constant, one close to the reported magnetite bulk value of magnetite $\left(40 \mathrm{~kJ} / \mathrm{m}^{3}\right)$, and the other one about 40 times smaller. As the figure shows, SAR values increase monotonically with the particle size. This trend is rather different from the one predicted by linear response theory, the predictions of which are also shown in the same figure, which predicts for high $K_{V}$ values a maximum SAR value, while our simulation results do not show any decrease with increasing particle size. ${ }^{[11]}$ This indicates that large particles with high $K_{V}$ values do not follow the predictions of linear response theory. Low $K_{V}$ values lead to much lower heat generation, which is in accordance with LRT in the frequency range investigated.

The results of the simulations of single particles and clusters as a function of frequency are shown in Fig. 2. SAR values calculated for single particles with a radius of $10 \mathrm{~nm}$ and $K_{V}=40 \mathrm{~kJ} / \mathrm{m}^{3}$, and of dumbbells, small clusters and large clusters made of the same particles. One can observe that simulations predict that the SAR values for particles are larger than those of clusters. As the cluster size increases, the SAR values decrease, with large clusters showing insignificant amount of heat generated compared to single particles. This 


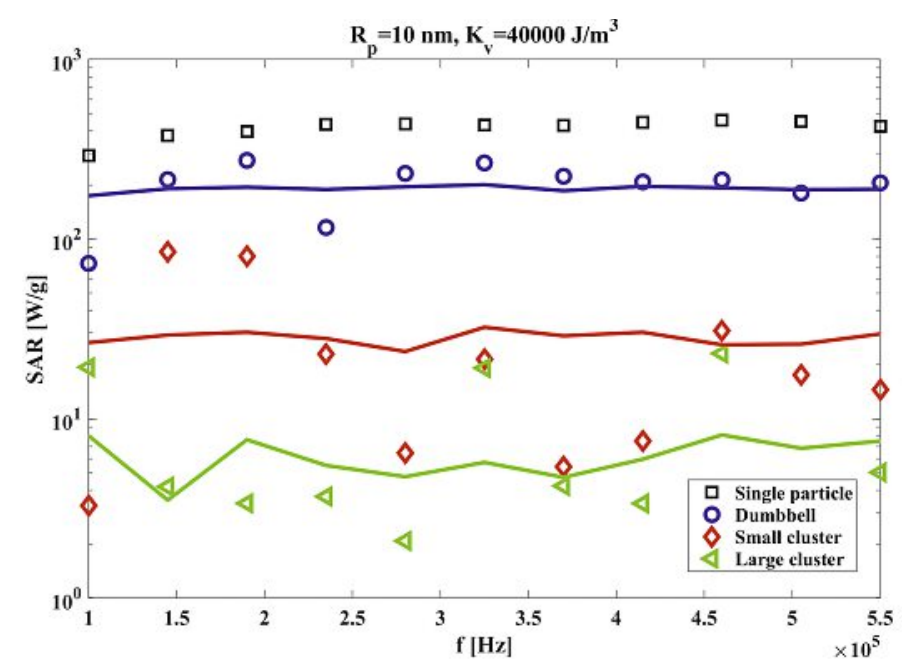

Fig. 2. SAR values of single SPIONs as a function of the frequency and of clusters, for the frequencies and magnetocrystalline anisotropy constants shown in the title. Points are the results of the Langevin simulations for particles and clusters, while lines are the predictions from simulations made with a single sphere with a viscosity increases by factor 2.38 (blue line), 17 (red line) and 73.5 (green line).

behavior can be well rationalized with the decreasing importance of Brownian relaxation as the cluster size increases. To quantify the effect of reducing the importance of Brownian relaxation, in Fig. 2 the SAR values for single particles immersed in a fluid with a viscosity 73.5, 17 and 2.38 times larger than that of water (which is the reference value), respectively, are also shown. An increase in viscosity by these factors has the same effect as the increase in the hydrodynamic volume due to the incorporation of particles in a large cluster, in a small cluster and in a dumbbell, respectively. The results show a reduction in SAR value comparable with the decrease in SAR values observed for large clusters, small clusters and dumbbells, respectively, which indicate that for such particles Brownian relaxation is the mechanism responsible for heat generation. Therefore, it is expected that the result should be completely different under two conditions: either in the case of clusters made of much smaller particles, or in the case of clusters made of particles with smaller $K_{V}$ values. In both cases, Brownian relaxation is not the dominant mechanism, but Neel relaxation becomes prevalent.

Fig. 3 shows data about SAR values obtained in the case of both clusters made of smaller particles $\left(6 \mathrm{~nm}\right.$ in radius) with $K_{V}=4 \cdot 10^{4} \mathrm{~J} /$ $\mathrm{m}^{3}$, and in the case of large particles $K_{V}=10^{3} \mathrm{~J} / \mathrm{m}^{3}$. Clusters made of small particles show SAR values smaller than those made of large particles, but it appears that clustering has almost no effect on the amount of heat that they can produce. A similar consideration can be made in the case of clusters made of particles with very low $K_{V}$ values, which also have very low SAR values. We have therefore performed simulations to find the conditions, i.e. the particle size and $K_{V}$ values, that maximize the values of SAR generated first by a particle, and then by a small cluster. The simulations indicate that, for particles, the optimal SAR values are reached with relatively large particles (12 nm in radius), which permit a substantial heat generation, and with medium value of the magnetocrystalline anisotropy constant $\left(K_{V} \sim 10^{4} \mathrm{~J} / \mathrm{m}^{3}\right)$. In the case of a cluster, the results are similar. This suggests that very large magnetocrystalline anisotropy constant values lead to a decrease in SAR value, because Neel relaxation is too slow, while Brownian relaxation is hampered by cluster formation. On the other hand, too small particles and too small magnetocrystalline anisotropy lead to too small heat losses.

\section{Conclusions}

To summarize, Langevin simulations have been performed to compute the heat generated by SPIONs, both individually dispersed and incorporated inside clusters. It was found that SPIONs

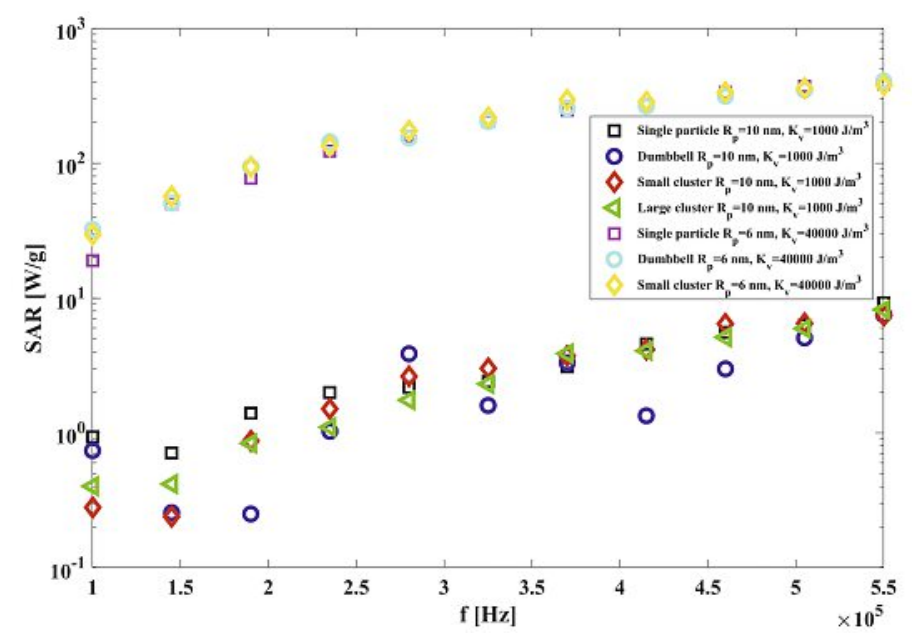

Fig. 3. SAR values of single SPIONs and clusters as a function of the frequency, for the cluster configurations, particle radius and magnetocrystalline anisotropy constants shown in the legend.

have the largest SAR values for large particle size, high frequency and high magnetocrystalline anisotropy. Brownian relaxation is the mechanism responsible for heat generation. Under these circumstances, however, the incorporation of particles in clusters significantly reduces the heat generation, by hampering Brownian relaxation. When Neel relaxation is the dominant relaxation mechanism for particles, clustering does not lead to significant differences in heat generation, which is however smaller than for particles with high magnetocrystalline anisotropy energy. The optimal configuration is therefore a compromise in particle size and magnetocrystalline anisotropy. These findings can help to better design hyperthermia formulation of SPIONs.

\section{Acknowledgements}

Financial support from the National competence center for research (NCCR) Bio-Inspired Materials is gratefully acknowledged.

Received: Nov. 29, 2018

[1] X. H. Huang, I. H. El-Sayed, W. Qian, M. A. El-Sayed, J. Am. Chem. Soc. 2006, 128, 2115, DOI: $10.1021 / \mathrm{ja} 057254 \mathrm{a}$.

[2] T. Neuberger, B. Schopf, H. Hofmann, M. Hofmann, B. von Rechenberg, $J$. Magn. Magn. Mater. 2005, 293, 483, DOI: 10.1016/j.jmmm.2005.01.064.

[3] A. K. Gupta, M. Gupta, Biomater. 2005, 26, 3995, DOI: 10.1016/j.biomaterials.2004.10.012.

[4] A. Figuerola, R. Di Corato, L. Manna, T. Pellegrino, Pharmacol. Res. 2010 62, 126, DOI: 10.1016/j.phrs.2009.12.012.

[5] J. Park, K. J. An, Y. S. Hwang, J. G. Park, H. J. Noh, J. Y. Kim, J. H. Park, N. M. Hwang, T. Hyeon, Nat. Mater. 2004, 3, 891, DOI: 10.1038/nmat1251.

[6] R. Massart, Ieee Trans. Magn. 1981, 17, 1247, DOI: 10.1109/ tmag.1981.1061188.

[7] A. Lak, D. Niculaes, G. C. Anyfantis, G. Bertoni, M. J. Barthel, S. Marras, M. Cassani, S. Nitti, A. Athanassiou, C. Giannini, T. Pellegrino, Sci. Rep. 2016, 6, DOI: 10.1038/srep33295.

[8] R. Fu, Y. Y. Yan, C. Roberts, Aip Adv. 2015, 5, DOI: 10.1063/1.4939514.

[9] C. A. Monnier, F. Crippa, C. Geers, E. Knapp, B. Rothen-Rutishauser, M. Bonmarin, M. Lattuada, A. Petri-Fink, J. Phys. Chem. C 2017, 121, 27164 , DOI: 10.1021/acs.jpcc.7b09094.

[10] J. Ge, Y. Hu, M. Biasini, W. P. Beyermann, Y. Yin, Angew. Chem. Int. Ed. 2007, 46, 4342, DOI: 10.1002/anie.200700197.

[11] R. E. Rosensweig, J. Magn. Magn. Mater. 2002, 252, 370, DOI: 10.1016/ s0304-8853(02)00706-0.

[12] R. Taukulis, A. Cebers, Phys. Rev. E 2012, 86, DOI: 10.1103/ PhysRevE.86.061405.

[13] E. C. Stoner, E. P. Wohlfarth, Phil. Trans. Royal Soc. London A 1948, 240 , 599, DOI: 10.1098/rsta.1948.0007.

[14] M. I. Shliomis, V. I. Stepanov, in 'Relaxation Phenomena in Condensed Matter', Vol. 87, Ed. W. Coffey, 1994, p. 1

[15] M. Lattuada, H. Wu, M. Morbidelli, J. Coll. Interf. Sci. 2003, 268, 96, DOI: 10.1016/j.jcis.2003.07.028

[16] M. Makino, M. Doi, J. Phys. Soc. Japan 2004, 73, 2739, DOI: 10.1143/ jpsj.73.2739. 